Treatment-naïve biopsies were analysed from seven TB patients, ten sarcoidosis patients and seven control samples. Bioinformatic analysis of RNAseq data identified one third of differentially expressed genes were communal to TB and sarcoidosis relative to control samples (absolute $\log _{2}$ fold change $\geq 1.5$, adjusted $\mathrm{P}$ value $<0.05$ ), with overlap of numerous pathways, including the extracellular matrix and cytokine signalling. Importantly, a TB unique cluster demonstrates TB results from a dysregulated inflammatory immune response, whereas a sarcoidosis predominant cluster relates to elevated lysosomal activity.

To translate these insights, we compared three primary human cell culture models: classical 2D; 3D alginate; and 3D collagen model. We demonstrated the Mtb-infected 3D collagen model most closely reflected clinical TB biopsies. We investigated signalling pathways shared between human disease and the 3D model, and used a systematic selection process to identify twelve intracellular enzymes as potential therapeutic targets. Sphingosine kinase 1 (SphK1) inhibition controlled $\mathrm{Mtb}$ growth in a dose-dependent manner, concurrently lowering intracellular $\mathrm{pH}$ in infected monocytes and suppressing inflammatory mediator secretion. Immunohistochemical staining confirmed that SphK1 is expressed in human lung TB granulomas, and therefore represents a potential novel hostdirected therapeutic target to improve TB outcomes.

\section{T4 REINFECTION WITH INFLUENZA A VIRUS LEADS TO RAPID CHANGES IN IMMUNOMODULATORY MOLECULES AND INFLAMMATORY SUBTYPES OF LUNG FIBROBLASTS AND EPITHELIAL CELLS}

${ }^{1} \mathrm{JC}$ Worrell, ${ }^{1} \mathrm{G}$ Finney, ${ }^{1} \mathrm{KE}$ Hargrave, ${ }^{1} \mathrm{C}$ Hansell, ${ }^{2} \mathrm{~J}$ Singh Nijjar, ${ }^{1} \mathrm{~F}$ Morton, ${ }^{1} \mathrm{~J} \mathrm{Cole}$, ${ }^{1}$ MKL MacLeod. 'Institute of Infection, Immunity and Inflammation, University of Glasgow, Glasgow, UK; ${ }^{2}$ Department of Medicine, University of Cambridge, Cambridge, UK

\subsection{6/thorax-2021-BTSabstracts.4}

Introduction and Objectives Influenza A virus (IAV) induces respiratory infections resulting in significant global mortality. Stromal cells are essential sources of chemokines and growth factors that promote immune cell survival and anti-viral responses. The concept that stromal cells are permanently altered by inflammatory responses is referred to as trained immunity. We hypothesise that IAV infections influence future immune responses via changes to lung stromal cells, enabling rapid communication with immune cells following subsequent infection.

Methods C57BL/6 mice were infected intranasally with IAV (WSN, 150PFU) for 30 days and subsequently re-challenged with IAV (X31, 200PFU) for 2 days. Mice were sacrificed at day $0,2,30$ and 32 post infection. A Nanostring assay was used to examine the transcriptional profiles of FACS sorted lung fibroblasts and epithelial cells. Differentially expressed genes were validated by qPCR and flow cytometry. The expression of upstream transcriptional drivers of these changes were compared with publicly available RNA-seq and ChIP-seq datasets. The potential impact of these changes on stromalimmune cell communication were assessed using immunohistochemistry and immunofluorescence.

Results Genes involved in $\mathrm{T}$ cell communication were significantly upregulated in lung fibroblasts (Cd274, Cxcl10) and epithelial cells (Tnfsf10, Icam2) following secondary IAV infection (d32), compared to $\mathrm{d} 30$. These changes were accompanied by elevated IFN-response genes (Bst2, Ifi47, Irf7).
Fibroblasts displayed enrichment in genes involved in biological processes regulating $\mathrm{T}$ cell activation, while epithelial cells were enriched for genes that regulate cytokines (IFN $\alpha / \beta$, $\mathrm{TNF} \alpha$ ). After secondary IAV infection, stromal cells rapidly upregulated genes involved in antigen processing and presentation (Tap1, Tapbp), compared to $\mathrm{d} 30$. The transcription factor SpiB was identified as a shared upstream regulator of these genes. Interestingly, in contrast to $\mathrm{d} 2$ post primary infection, MHCII+ epithelial cells were elevated following secondary infection, while the frequency of IFN-responsive fibroblasts was decreased. $\mathrm{T}$ and $\mathrm{B}$ cells were located near airway epithelial cells at $\mathrm{d} 30$ and were retained following secondary infection, indicating ongoing communication between these cells.

Conclusions Our data, show that lung fibroblasts and epithelial cells can display overlapping and functionally discrete responses to IAV infection, enabling them to rapidly communicate with lung $\mathrm{T}$ cells following a subsequent infection.

Please refer to page A188 for declarations of interest related to this abstract.

\section{T5 RESPIRATORY PARTICLE AND DROPLET EMISSION DURING SPEECH AND EXERCISE}

${ }^{1,2,3} \mathrm{CM}$ Orton, ${ }^{4} \mathrm{HE}$ Symons, ${ }^{1} \mathrm{~B}$ Moseley, ${ }^{4} \mathrm{~J}$ Archer, ${ }^{5} \mathrm{NA}$ Watson, ${ }^{1,3} \mathrm{KEJ}$ Philip, ${ }^{6} \mathrm{~B}$ SaccenteKennedy, ${ }^{7}$ DAE Costello, ${ }^{8} \mathrm{WJ}$ Browne, ${ }^{9,10} \mathrm{JD}$ Calder, ${ }^{4} \mathrm{BR}$ Bzdek, ${ }^{1,11} \mathrm{JH}$ Hull, ${ }^{4} \mathrm{JP}$ Reid,

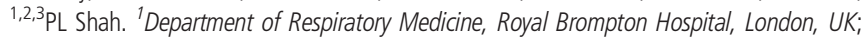
${ }^{2}$ Department of Respiratory Medicine, Chelsea and Westminster Hospital, London, UK; ${ }^{3}$ National Heart and Lung Institute, Imperial College London, London, UK; ${ }^{4}$ School of Chemistry, University of Bristol, Bristol, UK; ${ }^{5}$ Department of Ear, Nose and Throat Surgery, Guys and St. Thomas NHS Foundation Trust, London, UK; ${ }^{6}$ Speech and Language Therapy Department, Royal National Ear Nose and Throat Hospital, London, UK; ${ }^{\top}$ Ear, Nose and Throat Department, Wexham Park Hospital, Slough, UK; ${ }^{8}$ School of Education, University of Bristol, Bristol, UK; ${ }^{9}$ Department of Bioengineering, Imperial College London, London, UKi ${ }^{10}$ Fortius Clinic, London, UK; ${ }^{11}$ Institute of Sport, Exercise and Health (ISEH), UCL, London, UK

\subsection{6/thorax-2021-BTSabstracts.5}

Background The coronavirus disease-19 (COVID-19) pandemic has profoundly impacted sports and exercise, disrupting a plethora of events worldwide. Aerosol transmission is increasingly recognised as an important route for severe acute respiratory syndrome coronavirus 2 (SARS-CoV-2), with systematic evaluation of particulate matter release during exercise required to understand and mitigate transmission risk.

Methods Healthy participants $(n=25)$ performed a two stepped, flat-wave cardiopulmonary exercise test (CPET) on a cycle ergometer to replicate vigorous exercise (80\% of anaerobic workload) and high intensity exercise (anaerobic workload $+30 \%$ of the difference between anaerobic workload and peak workload), as determined by a maximally exhaustive CPET performed one hour previously. Concurrent measurements of aerosol and ventilatory data were recorded via a sampling line connected to an aerodynamic particle sizer (APS). Further synchronous data were collected at rest and when speaking at 70-80dBA. Droplet data were collected at rest and during high intensity exercise, using water sensitive paper.

Findings Median aerosol number concentration during speaking (at $70-80 \mathrm{dBA}), 0 \cdot 26 \mathrm{~cm}^{-3}$, was greater than during vigorous exercise, $0.12 \mathrm{~cm}^{-3}(\mathrm{p}<0.001)$ but not different to high intensity exercise, $0.24 \mathrm{~cm}^{-3}(\mathrm{p}=0 \cdot 92)$. Median aerosol mass concentration during speaking, $0.40 \mu \mathrm{g} / \mathrm{m}^{-3}$, was greater than during vigorous exercise $\left(0 \cdot 17 \mu \mathrm{g} / \mathrm{m}^{-3}, \mathrm{p}<0 \cdot 001\right)$, but not different to 